\title{
Protostar formation in the early universe
}

\author{
Naoki Yoshida ${ }^{1}$ \\ ${ }^{1}$ Department of Physics, Nagoya University \\ Furocho, Chikusa Nagoya 464-8602, Japan \\ email: nyoshida@a.phys.nagoya-u.ac.jp
}

\begin{abstract}
We study the formation of primordial proto-stars in a $\Lambda$ CDM universe using ultra high-resolution cosmological simulations. Our approach includes all the relevant atomic and molecular physics to follow the thermal evolution of a prestellar gas cloud to "stellar" densities. We describe the numerical implementation of the physics. We also show the result of a simulation of the formation of primordial stars in a reionized gas.
\end{abstract}

Keywords. cosmology: theory, molecular processes, radiative transfer

\section{Introduction}

The study of primordial star formation has a long history. The formation of the first cosmological objects via gas condensation by molecular hydrogen cooling has been studied for many years since the late 1960's. One-dimensional hydrodynamic simulations of spherical gas collapse were performed by a number of researchers (Omukai \& Nishi 1998), including a detailed treatment of all the relevant chemistry and radiative processes and thus were able to provide accurate results on the thermal evolution of a collapsing primordial gas cloud up to stellar densities. These authors found that, while the evolution of a spherical primordial gas cloud proceeds in a roughly self-similar manner, there are a number of differences in the thermal evolution from that of present-day, metal- and dust-enriched gas clouds.

Recently, three-dimensional hydrodynamic calculations were performed by several groups. Statistical properties of primordial star-forming clouds and the overall effect of cosmological bias have been studied in detail by Yoshida et al. (2003) and Gao et al. (2007). Simulations of the formation of primordial proto-stars have been hampered by complexity of physics such as radiative transfer in a very high-density prestellar gas. A critical technique we describe in this contribution is computation of molecular line opacities and continuum opacities. With the implementation of optically thick cooling, the gas evolution can be accurately followed to much higher densities than was possible in the previous studies (Yoshida et al. 2006). We show that the method works well in problems of collapsing gas clouds, in terms of accurate computation of radiative cooling rates and of resulting density and temperature structure. We apply this technique to a cosmological simulation of the formation of primordial star.

\section{Cosmological simulation}

We follow the standard procedure to set up cosmological initial conditions for a flat $\Lambda$ Cold Dark Matter cosmology with $\Omega_{\Lambda}=0.7, \Omega_{\text {darkmatter }}=0.26, \Omega_{\text {baryon }}=0.04$, and $H_{0}=70 \mathrm{~km} / \mathrm{sec} / \mathrm{Mpc}$. We use a multi-scale re-simulation technique to achieve a high mass resolution in the region where non-linear objects are formed early. The simulation volume has a side-length of 200 comoving kilo-parsecs. By employing a threelevel refinement to this volume, we achieve an initial mass resolution of $M_{\mathrm{dm}}=0.135 M_{\odot}$ 
and $M_{\mathrm{gas}}=0.021 M_{\odot}$ in the high-density region. We evolve the system until a first star-forming gas cloud is formed in one of the dark matter halos in the high-resolution region.

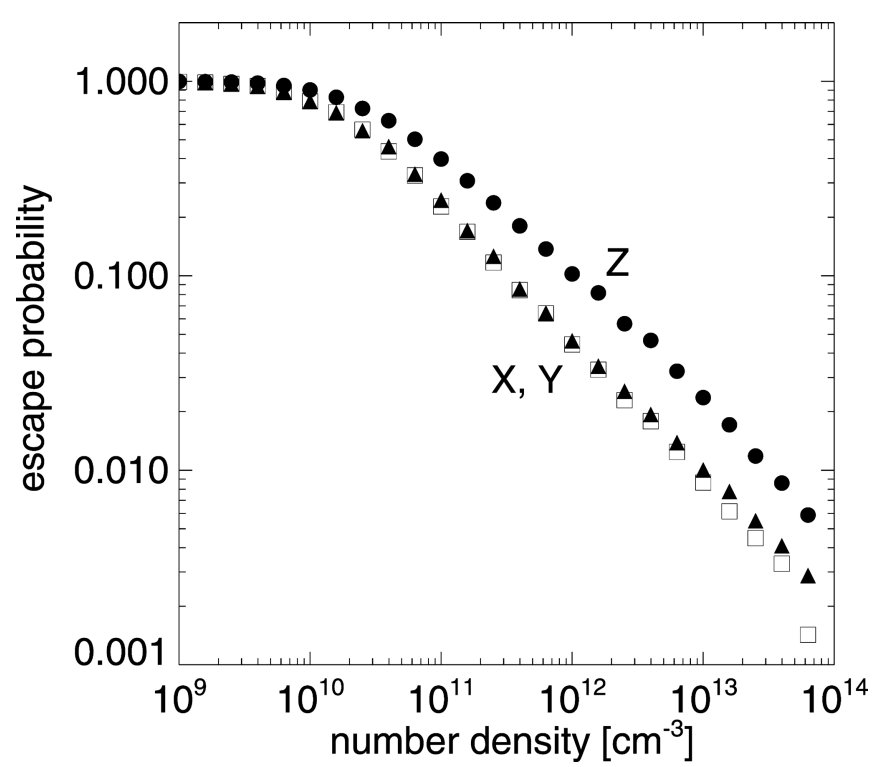

Figure 1. The escape probability for a line photon.

\section{Radiative transfer}

\subsection{Molecular hydrogen line cooling}

A primordial gas can cool radiatively by molecular hydrogen line cooling at low densities. When the gas density and the molecular fraction are high, the cloud becomes opaque to molecular lines and then $\mathrm{H}_{2}$ line cooling becomes inefficient. The net cooling rate can be expressed as

$$
\Lambda_{\mathrm{H}_{2}, \text { thick }}=\sum_{u, l} h \nu_{u l} \beta_{\mathrm{esc}, u l} A_{u l} n_{u},
$$

where $n_{u}$ is the population density of hydrogen molecules in the upper energy level $u$, $A_{u l}$ is the Einstein coefficient for spontaneous transition, $\beta_{\mathrm{esc}, u l}$ is the probability for an emitted line photon to escape without absorption, and $h \nu_{u l}=\Delta E_{u l}$ is the energy difference between the two levels.

In order to calculate the escape probability, we first evaluate the opacity for each molecular line as

$$
\tau_{l u}=\alpha_{l u} L
$$

where $L$ is the characteristic length scale. Since the absorption coefficients $\alpha_{l u}$ are computed in a straightforward manner, although somewhat costly, the remaining key task is the evaluation of the length scale $L$. By noting that the important quantity we need is the effective gas cooling rate, we can formulate a reasonable and well-motivated approximation. To this end, we decided to use the Sobolev method that is widely used in the study of stellar winds and planetary nebulae. 
We calculate the Sobolev length along a line-of-sight as

$$
L_{r}=\frac{v_{\text {thermal }}}{\left|d V_{r} / d r\right|},
$$

where $v_{\text {thermal }}=\sqrt{k T / m_{\mathrm{H}}}$ is the thermal velocity of $\mathrm{H}_{2}$ molecules, and $V_{r}$ is the fluid velocity in the direction. A suitable angle-average must be computed in order to obtain the net escape probability. Details are found in Yoshida et al. (2006).

In Fig. 1, we show the direction-dependence of the escape probabilities for one of the strongest $\mathrm{H}_{2}$ lines. We use an output of our high-resolution cosmological simulation at the time when the central density is $n_{\mathrm{c}}=10^{14} \mathrm{~cm}^{-3}$. To make this plot, we configure the coordinate such that the $z$-direction is aligned to the angular momentum vector of the central $1 M_{\odot}$ portion. The central part cools rapidly by $\mathrm{H}_{2}$ lines and flattens slightly, to have a prolate shape. The velocity gradient in $z$-direction is larger than those in the other two orthogonal directions, and hence the molecular lines preferentially escape in the $z$-direction.

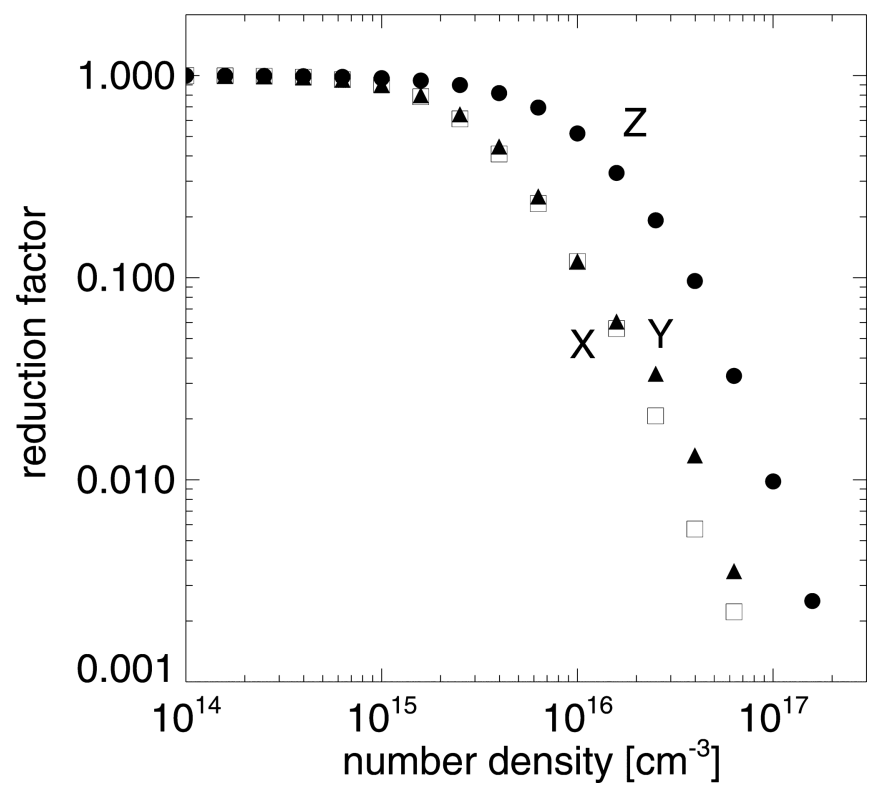

Figure 2. The ratio of $\Lambda_{\mathrm{CIE}, \text { thick }} / \Lambda_{\mathrm{CIE} \text {,thin }}$ as a function of density. We use an output when the central density reaches $10^{20} \mathrm{~cm}^{-3}$.

\subsection{Cooling by collision-induced emission}

At densities greater than $n \sim 10^{14} \mathrm{~cm}^{-3}$, hydrogen molecules collide with each other very frequently, making it possible to interact with photons by induced electric dipoles. This process is known as collision-induced emission (CIE), the opposite process to collisioninduced absorption:

$$
\begin{aligned}
\mathrm{H}_{2}(v, J)+\mathrm{H}_{2} & \rightarrow \mathrm{H}_{2}\left(v^{\prime}, J^{\prime}\right)+\mathrm{H}_{2}+h \nu, \\
\mathrm{H}_{2}(v, J)+\mathrm{He} & \rightarrow \mathrm{H}_{2}\left(v^{\prime}, J^{\prime}\right)+\mathrm{He}+h \nu . \\
\mathrm{H}_{2}(v, J)+\mathrm{H} & \rightarrow \mathrm{H}_{2}\left(v^{\prime}, J^{\prime}\right)+\mathrm{H}+h \nu . \\
\mathrm{H}+\mathrm{He} & \rightarrow \mathrm{H}+\mathrm{He}+h \nu .
\end{aligned}
$$


These processes yield very complex spectra, and have an essentially continuum appearance. We calculate the total emissivity by integrating the contribution from each transition:

$$
\eta_{\mathrm{CIE}}=\frac{2 h \nu^{3}}{c^{2}} \sigma_{\mathrm{CIE}} n\left(\mathrm{H}_{2}\right) \exp \left(-\frac{h \nu}{k T}\right) .
$$

We use the updated cross-sections $\sigma_{\text {CIE }}$ for $\mathrm{H}_{2}-\mathrm{H}_{2}, \mathrm{H}_{2}-\mathrm{H}, \mathrm{H}_{2}-\mathrm{He}$, and $\mathrm{H}-\mathrm{He}$ collisions.

Next, we implement computation of local optical depth to continuum radiation using the Planck opacity table of Lenzuni et al. (1991). The continuum opacity is used to evaluate the net CIE cooling rate. We calculate local optical depths in six orthogonal directions from a target point in a smoothed particle hydrodynamics manner (see, e.g., Yoshida et al. 2007), by projecting the cubic-spline kernels of surrounding gas particles which have their own densities and temperatures. The net energy transfer rate in each direction scales as $\Lambda \propto 1 /(1+\tau)$ when the optical depth is small, whereas, for large optical depths, it should scale as $\Lambda \propto 1 / \tau^{2}$. In practice we adopt a simple double power law:

$$
f=\frac{1}{(1+\tau)(1+(\tau / 10))}
$$

which approximates the above two conditions at small and large optical depths. In order to perform a suitable angle averaging, we take the mean of six directions,

$$
f_{\text {reduce }}=\frac{1}{6} \sum f_{i}
$$

Fig. 2 shows the reduction factor for the CIE cooling rate against local density. We note that, although our method is still approximate, it takes into account local density, temperature, and their structures (geometry) in a self-consistent manner. Our method can be applied to general problems, whereas simple functional fits for cooling rates that are based only on local density will fail in estimating the true cooling rate when the cloud core is prolate and/or has a complex structure such as in a (weak) turbulent velocity field.

\section{Primordial protostar}

We define a constant density, atomic gas core as a protostar that is pressure-supported. At the final output time, a protostar formed with a mass of just 0.01 solar masses. It had an initial radius of $\sim 5 \times 10^{11} \mathrm{~cm}$, similar to that of present-day protostars in theoretical calculations. The central particle number density of the protostar was $\sim 10^{21} \mathrm{~cm}^{-3}$ and the temperature was well above 10,000 K. Details of the formation process of this protostar is found in Yoshida, Omukai, \& Hernquist (2008).

The protostar has an atomic core with mass $0.01 M_{\odot}$ within a fully molecular part of $\sim 1 M_{\odot}$. At the time of protostar formation, the central temperature was so high that almost all the molecules are now collisionally dissociated within an enclosed mass of $0.01 M_{\odot}$. The structure is similar to those found in earlier one-dimensional spherically symmetric calculations (e.g. Ripamonti et al. 2002).

\section{Primordial star-formation in a reionized gas}

We employ the technique described in the previous sections in another cosmological simulation. Earlier in Yoshida et al. (2007), we used a large cosmological simulation to study the evolution of early relic HII regions until second-generation gas clouds are formed. We further explore the evolution of these prestellar gas clouds. The highest density achieved by the simulation is $\sim 10^{18} \mathrm{~cm}^{-3}$, at which point the central core is 


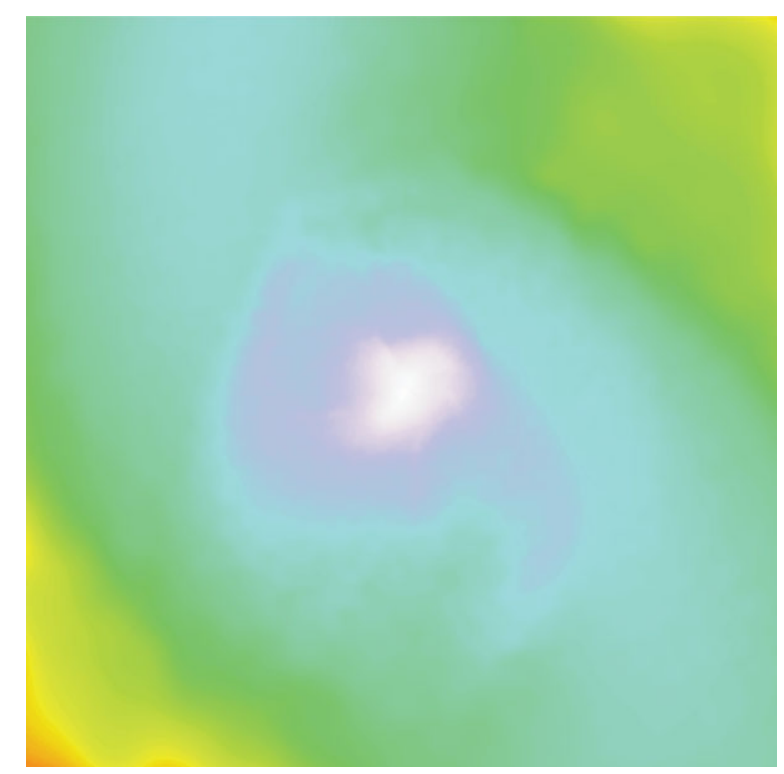

Figure 3. The newly born protostar in our simulation. We show the projected mean density-weighted temperature. The shown region has a side length of 25 astronomical units. The central part has a temperature of $\sim 12,000 \mathrm{~K}$.

optically thick even to continuum. Full-scale dissociation of hydrogen molecules is taking place in the core, which works as an effective cooling mechanism. We investigate in detail the structure of such gas clouds. We then compute the gas mass accretion rate and use it as an input to a proto-stellar calculation.

The temperature structure around the second-generation star can be understood by appealing to various atomic and molecular processes. HD line cooling brings the gas temperature below 100 Kelvin. Note that the minimum temperature is set by that of the cosmic microwave background.

The central proto-stellar 'seed' is accreting the surrounding gas at a rate $>10^{-3} M_{\odot} / \mathrm{yr}$ and thus a star with mass $\sim 10 M_{\odot}$ will form within $10^{4}$ years. However, the final stellar mass is determined by processes such as radiative feedback from the protostar. We treat the evolution of a protostar as a sequence of a growing hydrostatic core with an accreting envelope. The ordinary stellar structure equations are applied to the hydrostatic core. The structure of the accreting envelope is calculated under the assumption that the flow is steady for a given mass accretion rate.

Fig. 4 shows the resulting evolution of the protostar. After a transient phase and an adiabatic growth phase at $M_{*}<10 M_{\odot}$, the protostar enters the Kelvin-Helmholtz phase and contracts by radiating its thermal energy. When the central temperature reaches $10^{8} \mathrm{~K}$, hydrogen burning by the CNO cycle begins with a slight amount of carbon synthesized by helium burning. This phase is marked by a solid circle in the figure. The energy generation by hydrogen burning halts contraction when the mass is $35 M_{\odot}$ and its radius is $\sim 2.8$ solar radii. Soon after, the star reaches the zero-age main sequence (ZAMS). The protostar relaxes to a ZAMS star within about $10^{5}$ years from the birth of the protostellar seed. Accretion is not halted by radiation from the protostar to the end of our calculation.

It is important to point out that the mass of the parent cloud from which the star formed is $M_{\text {cloud }} \sim 40 M_{\odot}$. The final stellar mass is likely limited by the mass of the 


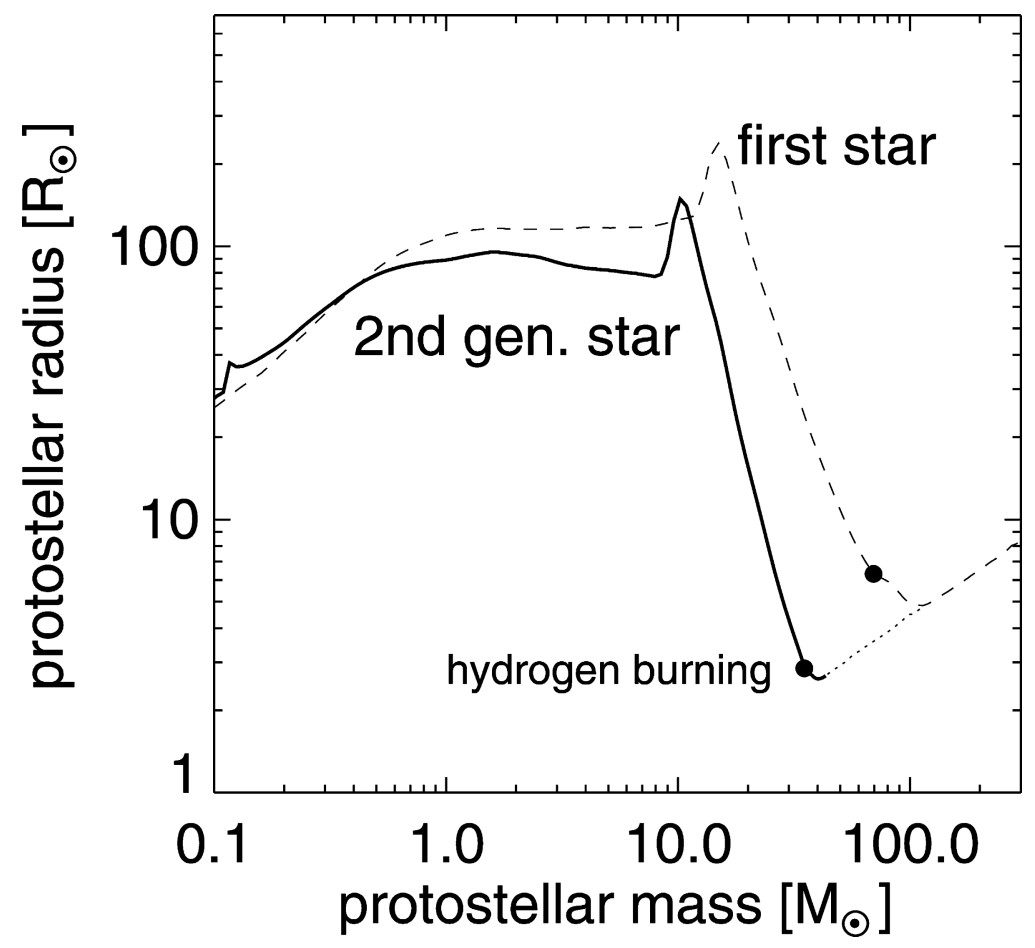

Figure 4. Evolution of the proto-stellar radius and the mass (solid line). The solid circle marks the time when efficient hydrogen burning begins. The dotted line shows the mass (and radius) growth which is calculated under the assumption that a larger amount of gas than the parent cloud can be accreted. For reference we also show the result from Y06 for a first generation star that forms in an initially neutral gas cloud.

gravitationally unstable parent cloud. We thus argue that primordial stars formed from an ionized gas are massive, with a characteristic mass of several tens of solar masses, allowing overall uncertainties in the accretion physics and also the dependence of the minimum gas temperature on redshift. They are smaller than the first stars formed from a neutral gas, but are not low-mass objects as suggested by earlier studies.

The elemental abundance patterns of recently discovered hyper metal-poor stars suggest that they might have been born from the interstellar medium that was metal-enriched by supernovae of these massive primordial stars.

\section{References}

Omukai, K. \& Nishi, R. 1998, ApJ, 508, 141

Yoshida, N., Abel, T, Hernquist, L., \& Sugiyama, N., 2003, ApJ, 592, 645

Gao, L., Yoshida, N., Abel, T., Frenk, C. S., Jenkins, A., \& Springel, V., 2007, MNRAS, 378, 449

Yoshida, N., Omukai, K., Hernquist, L., \& Abel, T., 2006, ApJ, 652, 6 (Y06)

Yoshida, N., Oh, S.-P., Kitayama, T., \& Hernquist, L., 2007, ApJ, 663, 687

Lenzuni, P., D., Chernoff, D. F., \& Salpeter, E. E., 1991, ApJS, 76, 759

Yoshida, N., Omukai, K., \& Hernquist, L., 2007, ApJL, 667, 117

Yoshida, N., Omukai, K., \& Hernquist, L., 2008, Science, 321, 669

Ripamonti, E., Haardt, F., Ferrara, A., \& Colpi, M., 2002. MNRAS, 334, 401 